\title{
Boundaries of a systemic disease: a protean presentation of giant cell arteritis
}

\author{
António Mesquita, ${ }^{1,2}$ Lara Camara, ${ }^{1}$ Catarina Patrício, ${ }^{1}$ Vítor Brotas ${ }^{1}$
}

${ }^{1}$ Internal Medicine, Hospital Santo António dos Capuchos, Lisboa, Portugal

${ }^{2}$ Pathophysiology Department, NOVA Medical School, Lisboa, Portugal

Correspondence to Dr António Mesquita; aefpmesquita@gmail.com

Accepted 16 March 2020

Check for updates

(c) BMJ Publishing Group

Limited 2020. No commercial re-use. See rights and permissions. Published by BMJ.

To cite: Mesquita $A$,

Camara L, Patrício C,

et al. BMJ Case Rep

2020;13:e232234.

doi:10.1136/bcr-2019-

232234

\section{SUMMARY}

A 60-year-old man was hospitalised with persistent fever, arm pain, dry cough and cholestasis. Diagnostic workup was remarkable for elevated inflammatory markers. Infectious diseases and autoimmune screening were negative. Imaging modalities excluded a neoplastic aetiology. Liver biopsy was negative for granulomatous or lymphomatous infiltrations. Giant cell arteritis (GCA) was suspected, but temporal artery Doppler ultrasound and biopsy were non-diagnostic. A positron emission tomography scan showed intense metabolic uptake in large vessels suggesting the diagnosis of GCA. Prednisolone was initiated with clinical and analytical improvement. At 1-year follow-up, there were no relapses and the patient remains symptom free.

\section{BACKGROUND}

Giant cell arteritis (GCA) is the most common systemic vasculitis. We think of it reflexively in older patients presenting with new-onset headache or acute vision loss. We know the common overlap between GCA and polymyalgia rheumatica (PMR). However we are less aware of its possible systemic presentation(s): fever of unknown origin (FUO), cough, cholestasis or limb claudication.

Temporal artery biopsy or Doppler ultrasound are giving way to other imaging modalities (CT, MRI or PET scan) that can show early signs of large vessel vasculitis (LVV).

\section{CASE PRESENTATION}

A previously healthy 60 -year-old man was admitted to the emergency department of another hospital with a 1 week history of pain in the medial aspect of both forearms, not related to physical activity. Acute coronary syndrome and aortic dissection were ruled out. An orthopaedic surgeon requested a cervical spine CT scan to evaluate possible radicular nerve compression, which showed no significant findings. A non-steroid anti-inflammatory drug (NSAID) was prescribed, resulting in pain improvement. During the following 2 weeks, the patient reported daily high fever $\left(39^{\circ} \mathrm{C}-40^{\circ} \mathrm{C}\right)$ and dry cough. He was re-evaluated in the same hospital and admission was proposed due to elevated liver enzymes, assuming an NSAID-induced toxic hepatitis. The patient refused and sought us for a second opinion.

The patient denied night sweats, weight loss, newonset headache, visual disturbances, jaw claudication, rhizomelic pain, limb claudication, sputum, dyspnoea, pleuritic chest pain, abdominal pain, nausea, vomiting, choluria or acholia. He reported no recent travelling, animal contacts, recreational drug use or sexual risk behaviour.

Clinical examination was unremarkable, except for discrete rales in both lung fields. There was no tenderness or temporal artery thickening, arterial bruits, lymphadenopathies, skin rash, heart murmurs, neurologic deficits, liver or spleen enlargement.

During the hospital stay, daily high fever and dry cough persisted, until the diagnosis was reached.

\section{INVESTIGATIONS}

- Blood analysis: normocytic normochromic anaemia (haemoglobin $10.4 \mathrm{~g} / \mathrm{L}$, mean globular volume $88 \mathrm{fL}$, mean globular haemoglobin $31.2 \mathrm{pg}$ ), increased inflammation markers (leukocyte count $12 \times 10^{9} / \mathrm{L}$ with $87 \%$ neutrophils, C-reactive protein (CRP) $-385 \mathrm{mg} / \mathrm{L}$, erythrocyte sedimentation rate (ESR)$110 \mathrm{~mm} /$ hour, platelet count $733 \times 10^{9} / \mathrm{L}$, fibrinogen $9.0 \mathrm{~g} / \mathrm{L}$, ferritin $6591 \mathrm{ng} / \mathrm{mL}$ ).

Aspartate aminotransferase (AST) $85 \mathrm{U} / \mathrm{L}$, alanine aminotransferase (ALT) $189 \mathrm{U} / \mathrm{L}$, alkaline phosphatase (ALP) 316U/L, gammaglutamyl transpeptidase (GGT) $203 \mathrm{U} / \mathrm{L}$, total bilirubin $0.50 \mathrm{mg} / \mathrm{dL}$, lactate dehydrogenase $300 \mathrm{U} / \mathrm{L}$.

Creatinine $0.90 \mathrm{mg} / \mathrm{dL}$, sodium $140 \mathrm{mEq} / \mathrm{L}$, potassium $4.1 \mathrm{mEq} / \mathrm{L}$.

Thyroid stimulating hormone $1.81 \mu \mathrm{UI} / \mathrm{mL}$, free thyroxine $0.93 \mathrm{ng} / \mathrm{dL}$. Triglycerides $78 \mathrm{mg} /$ dL.

Serum protein electrophoresis: alfa-1 globulins $8.9 \mathrm{~g} / \mathrm{L}$ (normal range 1.8-4.1), alfa-2 globulins $14.9 \mathrm{~g} / \mathrm{L}$ (normal range 4.5-9.8) with normal beta and gamma globulin values.

Normal urine sample.

- Antinuclear antibodies, anti-neutrophil cytoplasmic antibodies, complement and rheumatoid factor: negative.

- Serology for HIV, hepatitis virus (A, B and C), cytomegalovirus, Epstein-Barr virus, parvovirus, syphilis, Borrelia, Brucella, Coxiella, Rickettsia, Chlamydia, Mycoplasma: negative. Interferon-gamma releasing assay: negative.

- Blood and urine cultures: negative.

- Transthoracic echocardiogram (TTE): normal.

- Temporal artery Doppler ultrasonography (DUS): normal.

- Ophthalmoscopic examination: normal.

- Thoraco-abdominopelvic CT scan: normal. 


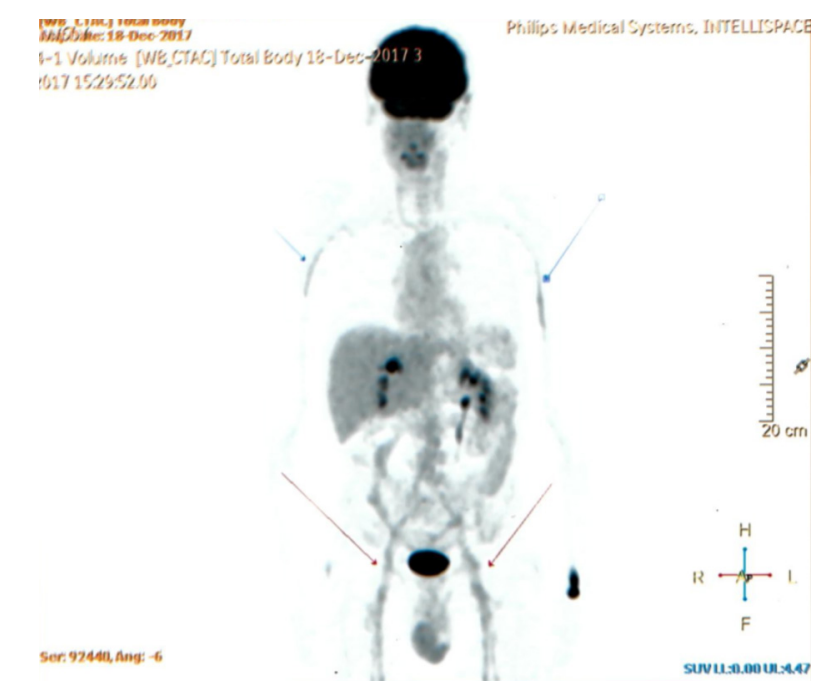

Figure 1 Positron emission tomography scan showing intense diffuse hypermetabolic fluorodeoxyglucose uptake in the abdominal aorta (SUVmax 3.64) and common iliac (SUVmax 3.60), femoral (SUVmax 3.60) and humeral arteries (SUVmax 3.50), bilaterally. Less evident uptake in the aortic arch. Threshold used was the liver - mean SUV 2.50 .

- Bone marrow biopsy, aspirate and mycobacterial culture: normal.

- Liver biopsy - direct examination, nucleic acid amplification test and culture for Mycobacterium tuberculosis: negative; histopathology: intrahepatocyte hemosiderin deposition with preferential distribution in the acinar zones 2 and 3 (grade 2 of Rowe).

- Temporal artery biopsy: normal.

- PET scan: intense diffuse hypermetabolic fluorodeoxyglucose (FDG) uptake in the abdominal aorta (maximum standardised uptake value - SUVmax - 3.64) and common iliac (SUVmax 3.60), femoral (SUVmax 3.60) and humeral arteries (SUVmax 3.50), bilaterally. Less evident uptake in the aortic arch. Threshold used was the liver - mean SUV 2.50 (figure 1).

\section{DIFFERENTIAL DIAGNOSIS}

Protracted fever syndrome fulfilling or not criteria for 'FUO' remains a considerable challenge for the clinician. ${ }^{1}$ The most prevalent causes of prolonged fever and FUO are infection, noninfectious inflammatory diseases and malignancy. Infections and malignancies as causes of FUOs have decreased over time, while inflammatory diseases and undiagnosed fevers have increased. ${ }^{2}$ Epidemiologic features, age and immunological status are relevant for diagnostic approach. Clinical and ancillary test findings suggesting a particular organ involvement should prompt a targeted investigation to eventually define the subjacent lesion. In our patient, three clinical clues were relevant, besides daily high fever: persistent dry cough, elevated liver enzymes and forearm pain.

The elevated inflammatory markers (ESR, CRP, ferritin, platelets) made infectious/inflammatory disease more likely than malignancy. Tuberculosis and Q fever - given our local epidemiology - as well as sarcoidosis were of concern due to their possible presentation with both lung and liver involvement. The normal CT scan, negative infectious diseases workup, unrevealing bone marrow biopsy and non- diagnostic liver biopsy excluded these aetiologies. Other less likely infectious diseases considered, solid tumours, and lymphoproliferative disorders were also deemed unlikely. Negative blood cultures and TTE were against infectious endocarditis.

Considering the high fever, markedly elevated ferritin and abnormal liver enzymes, hemophagocytic lymphohistiocytosis, a life-threatening entity, was considered. However, the patient did not meet the diagnostic criteria for this disease. Adult-onset Still's disease should also be regarded in the setting of marked increase in serum ferritin. Nonetheless, the presenting age and lack of classic findings such as rash, lymphadenopathy or serositis made this diagnosis less likely. Also, being a diagnosis of exclusion, other conditions had to be adequately investigated.

Forearm pain was the patient's initial complaint. The pain was persistent and not related to exertion. In another hospital, acute coronary syndrome, aortic dissection and radicular nerve compression were appropriately excluded. This symptom resolved after a 1 week course of NSAID. Initially overlooked, it acquired new meaning in the setting of systemic inflammation. Localising features are essential when approaching a patient with FUO. In our patient, arm pain could be representative of myositis, enthesitis or vascular inflammation. Taking into account the patient's age and the prominent ESR, a vasculitic process like GCA became the main diagnostic focus. The patient had no complaints reporting to temporal arteritis namely headache, visual disturbances or jaw claudication. Temporal artery ultrasound showed no abnormal findings and temporal artery biopsy was normal. CT, MRI and PET scans are suitable means to demonstrate large vessel inflammation. We chose PET scan because of its possible higher sensitivity in the earlier disease stage and because of the lack of expertise at our hospital centre for CT scan and MRI used in this setting. The PET scan confirmed intense diffuse hypermetabolic FDG uptake in the abdominal aorta and common iliac, femoral and humeral arteries, bilaterally, supporting the diagnosis of LVV. The presenting clinical features and diagnostic workup done allowed us to exclude other aetiologies of large-vessel vasculitis and aortitis such as Syphilis, Cogan's syndrome, Behçet's syndrome or spondyloarthropathies. The diagnosis of GCA was assumed.

\section{TREATMENT}

The patient was started on prednisolone $1 \mathrm{mg} / \mathrm{kg}$ (60 mg) per day and aspirin $150 \mathrm{mg} /$ day.

General health recommendations were provided, especially regarding corticoid adverse effects: blood pressure, glycaemic and weight control. Calcium and vitamin D supplementations were prescribed.

\section{OUTCOME AND FOLLOW-UP}

One month after starting treatment, a clinical and analytic improvement was observed. Fever and cough subsided and laboratory surrogate markers were normalised: haemoglobin $14.3 \mathrm{~g} / \mathrm{L}$, platelet count $240 \times 10^{9} / \mathrm{L}$, ESR $9 \mathrm{~mm} /$ hour, CRP $0.3 \mathrm{mg} / \mathrm{L}$, ferritin $730 \mathrm{ng} / \mathrm{mL}$, AST $20 \mathrm{U} / \mathrm{L}$, ALT $26 \mathrm{U} / \mathrm{L}$, ALP $107 \mathrm{U} / \mathrm{L}$ and GGT $31 \mathrm{U} / \mathrm{L}$.

At 1-year follow-up, he is asymptomatic with $5 \mathrm{mg}$ of prednisolone per day.

\section{DISCUSSION}

GCA was first described by Horton and colleagues in 1932 as a new form of arteritis that affected the temporal vessels. ${ }^{3}$ Historically, however, we find descriptions of this disease going back to X century Iraq, where Ophthalmologist Ali Ibn Isa excised and cauterised arteries to treat patients suffering from 'heat and 
inflammation of temporal muscles that sometimes ended in loss of vision'?

GCA is the most common idiopathic systemic vasculitis. ${ }^{4}$ Its incidence is directly related to age, almost never occurring before the age of 50 and rising steadily, thereafter, with over $80 \%$ of patients aged 70 years or older. ${ }^{5}$

GCA has been interchangeably referred to as temporal arteritis for decades. The idea that this disease presents mainly — or only - with cranial symptoms/signs permeates most of the literature and is evident in the American College of Rheumatology (ACR) classification criteria. ${ }^{6}$ However, the true systemic nature of CGA has been increasingly more recognised. The association with constitutional symptoms and PMR is well documented. Indeed, the close relationship with PMR goes beyond epidemiological observations; studies established a common pathophysiological base as patients with PMR show findings of vasculitis on imaging even without clinical signs and symptoms of GCA. ${ }^{7}$

Constitutional symptoms and elevated inflammatory markers in patients over 50 years of age are the hallmark features of GCA. In this setting, other manifestations, such as dry cough, can be unrecognised clues to the diagnosis. Cough was found in up to $13 \%$ of patients with GCA. ${ }^{8}$ Actual lung involvement is rare although some cases report multinodular pulmonary lesions and diffuse interstitial pattern. ${ }^{9}$ More often, dry cough leads to diagnostic confusion as it seems to arise from arterial vasculitis in the contiguity of the cough reflex pathway, instead of structural pulmonary lesions. ${ }^{10}$

Liver involvement is exceedingly rare in GCA, but cholestatic hepatitis has been reported. ${ }^{11} 12$ We were especially concerned about tuberculous hepatitis as well as other infiltrative processes. Liver biopsy, however, showed no granulomas or other pathologic changes suggestive of a diagnosis. Hepatic involvement associated with GCA has been shown to result from different histological changes such as bile stasis, fatty degeneration, portal tract inflammatory infiltration, granulomatous hepatitis or hepatic arteritis. ${ }^{11} 1314$ Notwithstanding, the biopsy performed on our patient revealed intrahepatocyte hemosiderin deposition. It is interesting to consider that the pivotal role of interleukin 6 (IL-6) in the pathogenesis of GCA helps us to understand this finding. By inducing the expression of Hepcidin, which in turn inhibits Ferroportin, IL- 6 promotes the sequestration of iron in reticuloendothelial cells. ${ }^{15}$ In our patient, intrahepatocyte hemosiderin deposition, in the setting of systemic inflammation, translated in a pattern of cholestatic hepatitis that was resolved with appropriate treatment. To the best of our knowledge, this is the first case report of GCA associated with hepatic hemosiderosis.

We are witnessing a shifting paradigm for GCA. We are looking at this disease as a clinical spectrum of three distinct phenotypes that can occur independently or can overlap each other: (1) cranial GCA; (2) extra-cranial, or large-vessel GCA and (3) PMR. ${ }^{16}$ This paradigm shift raises the question of diagnosis. The gold standard has been temporal artery biopsy but sensitivity of this procedure varies. On the one hand, the segmental nature of the arteritic involvement determines the existence of so-called 'skip lesions', affecting the rate of false-negative results. There is no unifying data supporting an optimal biopsy length. Studies and recommendations seem to point towards a standard length of at least $1 \mathrm{~cm}$, taking into account the post-fixation shrinkage of the specimen. ${ }^{1718}$ On the other hand, most cranial symptoms correlate poorly with the results of temporal artery biopsyonly jaw claudication and diplopia have been shown to have a strong positive predictive value. ${ }^{19}$ Extra-cranial GCA presents with different clinical manifestations and is associated with a bigger rate of false-negative results. A study of 74 patients with extra-cranial GCA reported that temporal artery biopsy findings were negative in $42 \%$ of patients. ${ }^{20}$ Knowing this, we decided to pursue temporal artery biopsy given the easier accessibility of this procedure compared with PET scan.

Interestingly, our patient did not meet the ACR diagnostic criteria for GCA. This is significant as to why we should look for imaging methods earlier in the diagnostic workup of GCA. Temporal and axillary artery DUS have been proposed as surrogates for biopsy; axillary artery DUS is routinely recommended if GCA or PMR is suspected, because temporal arteries may be spared in $40 \%$ of patients. ${ }^{21} 22$ In patients with suspected extra-cranial GCA, in whom temporal artery biopsy has lower sensitivity, DUS, computerised tomography angiography (CTA), magnetic resonance angiography (MRA) and PET scan have been shown to be of diagnostic worth. ${ }^{23}$ In fact, the European League Against Rheumatism already recognises imaging methods as diagnostic surrogates for GCA and recommends early imaging assuming high expertise and availability. ${ }^{24}$

PET scan has been studied as a diagnostic tool in vasculitis since the accidental finding of aortic FDG uptake during oncological staging. ${ }^{25}$ Increased FDG uptake serves as a marker of inflammation, translating the high glycolytic activity of inflammatory cells in the arterial walls. There has been discussion about how to best standardise diagnostic criteria for vasculitis using PET scan. Simple SUV metrics do not appear relevant in diagnosis given the interpatient variability in weight or FDG uptake in the blood. Some studies have used target to background ratio, expressed as the blood-normalised SUVmax. ${ }^{25}$ Similarly, evidence supports the use of a visual grading scale comparing vascular to liver uptake. The most recent consensus from the European Association of Nuclear Medicine and Society of Nuclear Medicine and Molecular Imaging recommends the use of a standardised 0 to 3 grading system for the diagnosis of LVV, whereas: $0=$ no uptake ( $\leq$ mediastinum); $1=$ low-grade uptake (<liver); $2=$ intermediate-grade uptake (=liver) and $3=$ high-grade uptake (>liver). ${ }^{26}$ Based on these recommendations, our patient has a grade 3 uptake, considered diagnostic of active LVV. Notably, our patient's PET scan did not show significant axillary artery uptake, which is one of the most common areas affected, particularly with humeral artery involvement.

Corticosteroids are the mainstay in the management of GCA. For PMR patients, initial treatment with low-dose corticosteroid is recommended, in most cases $15 \mathrm{mg}$ /day of prednisone (or equivalent). Cranial and extra-cranial GCA patients need higher doses, with the induction dose being higher for those presenting with threatened or established visual loss. ${ }^{27}$ Maintenance dose is generally $1 \mathrm{mg} / \mathrm{kg} / \mathrm{day}$ for $2-4$ weeks followed by gradual tapering. Given the high doses used, osteoporosis prevention is recommended. Pneumocystis jirovecii infection is a known complication of iatrogenic imunossupression. ${ }^{28}$ Although prevalence is low in patients only taking corticosteroids, prophylaxis should be contemplated. Because GCA commonly relapses when glucocorticoids are tapered, and considering the side effects related to their prolonged use, glucocorticoid sparing agents like tocilizumab or methotrexate are important management options. Tocilizumab, an interleukin- 6 inhibitor, has been shown to be an effective first-line option in achieving sustained glucocorticoidfree remission in patients with GCA. ${ }^{29}$

GCA is a disease of variable duration. Complete and sustained remission is possible with discontinuation of glucocorticoid therapy. However, relapses are common, occurring in more than $50 \%$ of patients, frequently with glucocorticoid therapy. ${ }^{30}$ Some patients may need to maintain low doses of prednisone for several years. Overall mortality is not affected, but screening 
for aortic aneurism is recommended in all patients in the 5 years following diagnosis. ${ }^{31}$

\section{Learning points}

- Giant cell arteritis (GCA) represents a clinical spectrum of three distinct phenotypes of cranial GCA, extra-cranial GCA and polymyalgia rheumatica.

- GCA should always be considered in the differential diagnosis of fever of unkown origin in patients over the age of 50 , even in the absence of classical symptoms of temporal arteritis.

- Liver involvement is exceedingly rare but can be a part of GCA's protean presentation due to direct hepatic artery involvement, granulomatous liver infiltration or systemic inflammation with hepatocyte hemosiderin deposition.

- Imaging modalities such as Doppler ultrasonography, magnetic resonance angiography, computerised tomography angiography and positron emission tomography scan need to be considered early in the diagnostic workup of GCA.

- Tocilizumab should be considered as an effective first-line option in achieving steroid-free remission.

Acknowledgements The authors would like to acknowledge Dr Pedro Ratão and Dr Francisco Norton Brandão, from the department of Nuclear Medicine of Instituto Português de Oncologia de Lisboa, for their expert and kind assistance with the discussion of the PET-CT scan findings.

Contributors $\mathrm{AM}$ and $\mathrm{LC}$ have contributed to the redaction of the article. CP and VB were responsible for reviewing the main document and advising on the writting process.

Funding The authors have not declared a specific grant for this research from any funding agency in the public, commercial or not-for-profit sectors.

Competing interests None declared.

Patient consent for publication Obtained.

Provenance and peer review Not commissioned; externally peer reviewed.

\section{REFERENCES}

1 Vanderschueren S, Knockaert D, Adriaenssens T, et al. From prolonged febrile illness to fever of unknown origin: the challenge continues. Arch Intern Med 2003;163:1033.

2 Horowitz HW. Fever of unknown origin or fever of too many origins? N Engl J Med 2013;368:197-9.

3 Henriet JP, Marin J, Gosselin J, et al. [The history of Horton's disease or... 10 centuries of a fascinating adventure]. J Mal Vasc 1989;14 Suppl C:93-7.

4 González-Gay MA, García-Porrúa C. Systemic vasculitis in adults in northwestern Spain, 1988-1997. Clinical and epidemiologic aspects. Medicine 1999;78:292-308.

5 Gonzalez-Gay MA, Vazquez-Rodriguez TR, Lopez-Diaz MJ, et al. Epidemiology of giant cell arteritis and polymyalgia rheumatica. Arthritis Rheum 2009;61:1454-61.
6 Abari SI. ACR revised criteria for early diagnosis of giant cell (temporal) arteritis. Autoimmune Dis Therapeutic Approach Open Access 2016;3:119-22.

7 Kermani TA, Warrington KJ. Advances and challenges in the diagnosis and treatment of polymyalgia rheumatica. Ther Adv Musculoskelet Dis 2014:6:8-19.

8 Zenone T, Puget M. Dry cough is a frequent manifestation of giant cell arteritis. Rheumatol Int 2013:33:2165-8.

9 Kramer MR, Melzer E, Nesher G, et al. Pulmonary manifestations of temporal arteritis. Eur J Respir Dis 1987;71:430-3.

10 Kondo T, Ohira Y, Uehara T, et al. Cough and giant cell arteritis. Int J Med 2018:111:747-8.

$11 \mathrm{Xu}$ J, Björnsson ES, Sundaram V. Severe cholestatic hepatitis due to large vessel vasculitis: report of two cases. Gastroenterol Rep 2018;6:gov061-71.

12 Towns K, Szmitko PE, Smith C, et al. It's not all in your head. N Engl J Med 2011;365:1329-34.

13 Pedro-Botet J, López MJ, Barranco C, et al. Granulomatous hepatitis and giant cell arteritis. Am J Gastroenterol 1994;89:1898-9.

14 Ogilvie AL, James PD, Toghill PJ. Hepatic artery involvement in polymyalgia arteritica. Clin Pathol 1981;34:769-72.

15 Wang C-Y, Babitt JL. Hepcidin regulation in the anemia of inflammation. Curr Opin Hematol 2016;23:189-97.

16 Dejaco C, Duftner C, Buttgereit F, et al. The spectrum of giant cell arteritis and polymyalgia rheumatica: revisiting the concept of the disease. Rheumatology 2017;56:506-15.

17 Dasgupta B, Borg FA, Hassan N, et al. BSR and BHPR guidelines for the management of giant cell arteritis. Rheumatology 2010;49:1594-7.

18 Ypsilantis E, Courtney ED, Chopra N, et al. Importance of specimen length during temporal artery biopsy. Br J Surg 2011;98:1556-60.

19 Smetana GW, Shmerling RH. Does this patient have temporal arteritis? JAMA 2002;287:92.

20 Brack A, Martinez-Taboada V, Stanson A, et al. Disease pattern in cranial and largevessel giant cell arteritis. Arthritis Rheum 1999;42:311-7.

21 Schmidt WA. Ultrasound in the diagnosis and management of giant cell arteritis. Rheumatology 2018;57:ii22-31.

22 Schmidt WA, Seifert A, Gromnica-Ihle E, et al. Ultrasound of proximal upper extremity arteries to increase the diagnostic yield in large-vessel giant cell arteritis. Rheumatology 2008;47:96-101.

23 Khan A, Dasgupta B. Imaging in giant cell arteritis. Curr Rheumatol Rep 2015;17.

24 Dejaco C, Ramiro S, Duftner C, et al. EULAR recommendations for the use of imaging in large vessel vasculitis in clinical practice. Ann Rheum Dis 2018;77:636-43.

25 Kemna MJ, Bucerius J, Drent M, et al. Aortic 18F-FDG uptake in patients suffering from granulomatosis with polyangiitis. Eur J Nucl Med Mol Imaging 2015;42:1423-9.

26 Slart RHJA, Writing group, Reviewer group. FDG-PET/CT(A) imaging in large vessel vasculitis and polymyalgia rheumatica: joint procedural recommendation of the EANM, SNMMI, and the PET Interest Group (PIG), and endorsed by the ASNC. Eur J Nucl Med Mol Imaging 2018;45:1250-69.

27 Hellmich B, Agueda A, Monti S, et al. 2018 update of the EULAR recommendations for the management of large vessel vasculitis. Ann Rheum Dis 2020;79:19-30.

28 Kermani TA, Ytterberg SR, Warrington KJ. Pneumocystis jiroveci pneumonia in giant cell arteritis: a case series. Arthritis Care Res 2011;63:761-5

29 Stone JH, Tuckwell K, Dimonaco S, et al. Trial of tocilizumab in giant-cell arteritis. N Engl J Med 2017;377:317-28.

30 Alba MA, García-Martínez A, Prieto-González S, et al. Relapses in patients with giant cell arteritis. Medicine 2014;93:194-201.

31 García-Martínez A, Arguis P, Prieto-González S, et al. Prospective long term follow-up of a cohort of patients with giant cell arteritis screened for aortic structural damage (aneurysm or dilatation). Ann Rheum Dis 2014;73:1826-32.

Copyright 2020 BMJ Publishing Group. All rights reserved. For permission to reuse any of this content visit

https://www.bmi.com/company/products-services/rights-and-licensing/permissions/

BMJ Case Report Fellows may re-use this article for personal use and teaching without any further permission.

Become a Fellow of BMJ Case Reports today and you can:

- Submit as many cases as you like

- Enjoy fast sympathetic peer review and rapid publication of accepted articles

- Access all the published articles

- Re-use any of the published material for personal use and teaching without further permission

Customer Service

If you have any further queries about your subscription, please contact our customer services team on +44 (0) 2071111105 or via email at support@bmj.com.

Visit casereports.bmj.com for more articles like this and to become a Fellow 